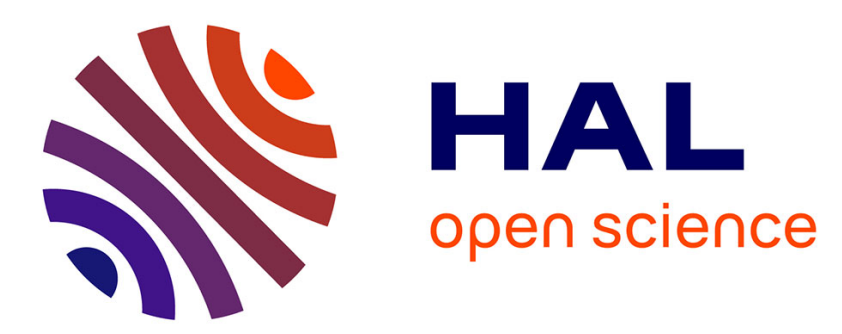

\title{
How familiarization and repetition modulate the picture naming network
}

Anaïs Llorens, Agnès Trébuchon, Stéphanie Riès, Catherine Liégeois-Chauvel, F.-Xavier Alario

\section{- To cite this version:}

Anaïs Llorens, Agnès Trébuchon, Stéphanie Riès, Catherine Liégeois-Chauvel, F.-Xavier Alario. How familiarization and repetition modulate the picture naming network. Brain and Language, 2014, 133, pp.47-58. 10.1016/j.bandl.2014.03.010 . hal-02097744

\section{HAL Id: hal-02097744 \\ https://hal.science/hal-02097744}

Submitted on 19 Nov 2019

HAL is a multi-disciplinary open access archive for the deposit and dissemination of scientific research documents, whether they are published or not. The documents may come from teaching and research institutions in France or abroad, or from public or private research centers.
L'archive ouverte pluridisciplinaire HAL, est destinée au dépôt et à la diffusion de documents scientifiques de niveau recherche, publiés ou non, émanant des établissements d'enseignement et de recherche français ou étrangers, des laboratoires publics ou privés.

\section{다(1)(2)}

Distributed under a Creative Commons Attribution - ShareAlikel 4.0 International 
Published in final edited form as:

Brain Lang. 2014 June ; 133: 47-58. doi:10.1016/j.bandl.2014.03.010.

\title{
How familiarization and repetition modulate the picture naming network
}

\author{
Anaïs Llorens ${ }^{1,2,{ }^{*}}$, Agnès Trébuchon ${ }^{1}$, Stéphanie Riès ${ }^{2,3}$, Catherine Liégeois-Chauvel ${ }^{1}$, and \\ F.-Xavier Alario ${ }^{2}$ \\ ${ }^{1}$ Institut des Neurosciences des systèmes \& Aix-Marseille Université \& INSERM UMR 1106 \\ ${ }^{2}$ Laboratoire de Psychologie Cognitive, Aix-Marseille Université \& CNRS UMR7290 \\ ${ }^{3}$ Helen Wills Neuroscience Institute, University of California, Berkeley
}

\begin{abstract}
A common strategy to reveal the components of the speech production network is to use psycholinguistic manipulations previously tested in behavioral protocols. This often disregards how implementation aspects that are nonessential for interpreting behavior may affect the neural response. We compared the electrophysiological (EEG) signature of two popular picture naming protocols involving either unfamiliar pictures without repetitions or repeated familiar pictures. We observed significant semantic interference effects in behavior but not in the EEG, contrary to some previous findings. Remarkably, the two protocols elicited clearly distinct EEG responses. These were not due to naming latency differences nor did they reflect a homogeneous modulation of amplitude over the trial time-window. The effect of protocol is attributed to the familiarization induced by the first encounter with the materials. Picture naming processes can be substantially modulated by specific protocol requirements controlled by familiarity and, to a much lesser degree, the repetition of materials.
\end{abstract}

\section{Keywords}

language production; speech; lexical access; semantic interference; blocked naming; familiarity; priming; electrophysiology

\section{Introduction}

Retrieving words from memory in order to express their thoughts is a key component of Humans' language production ability. According to the standard theoretical view, this process is one in which conceptual or semantic representations of the message to be

(C) 2014 Elsevier Inc. All rights reserved.

*Corresponding author: Anaïs Llorens Faculté de Médecine La Timone 27 Bd Jean Moulin Aile rouge $5^{\mathrm{e}}$ étage 13385 Marseille Cedex 05 France Tel: +33(0) 491384634 anais.1lorens@ univ-amu.fr.

Publisher's Disclaimer: This is a PDF file of an unedited manuscript that has been accepted for publication. As a service to our customers we are providing this early version of the manuscript. The manuscript will undergo copyediting, typesetting, and review of the resulting proof before it is published in its final citable form. Please note that during the production process errors may be discovered which could affect the content, and all legal disclaimers that apply to the journal pertain. 
conveyed drive the activation and selection of word-specific lexical representations (Caramazza \& Hillis, 1990; Damasio, Tranel, Grabowski, Adolphs, \& Damasio, 2004; Dell, Burger, \& Svec, 1997; Indefrey, 2011; Levelt, 1993; Levelt, 1992; Nickels, 2001). Laboratory research conducted to understand this process has made a widespread use of the picture naming task. Participants are presented with visual objects, usually one at a time, and they are asked to produce overtly the corresponding name (Alario et al., 2004; Bock, 1996; Glaser, 1992), an instruction that is presumed to trigger the activation of the semantic-tolexical pathway above-described.

The cortical network underlying visual object naming has been described in detail on the basis of functional imaging data, complemented with some temporal evidence from neurophysiological studies. Oral picture naming recruits a widely distributed network of cortical areas, predominantly located in the left hemisphere. The network starts with occipital and ventro-temporal structures. From $200 \mathrm{~ms}$ onwards, temporal structures are engaged in lexico-semantic processing (activation of the meaning of the picture and its possible names). Somewhat later, inferior parietal cortex and posterior temporal lobe are associated with phonological encoding. The left inferior frontal gyrus is thought to resolve conflict among alternative representations, as well as syllabification processes. Finally, bilateral pre-motor and motor areas, as well as the inferior frontal gyri, are engaged for articulatory planning and articulation (for review see Damasio et al., 2004; Indefrey \& Levelt, 2004; Indefrey, 2011; Llorens, Trébuchon, Liégeois-Chauvel, \& Alario, 2011; Price, 2012).

Regarding timing, perhaps the most influential spatio-temporal model of word production has been proposed by Indefrey (2011, and previous versions). This proposal has two specific features. First, it is directly based on a cognitive processing model (Levelt, Roelofs, \& Meyer, 1999) which has relatively strong modularity hypothesis, while alternative views have been defended (Rapp \& Goldrick, 2000). Second, and related, the model describes the stages that are deemed necessary, a priori, for producing a word. It does not consider activities or modulations that may occur collaterally and that may be detectable in brain signals (we shall come back to this point).

\section{Using brain activity to test behavioral protocols}

A strategy which is commonly used to reveal the components of this network is to test whether or not they are sensitive to psycholinguistic manipulations previously validated and interpreted in behavioral research (e.g. semantic or phonological relatedness; De Zubicaray \& McMahon, 2009; see Riès, Janssen, Burle, \& Alario, 2013for a different approach based on the timing of the events). In addition to such manipulations of theoretical interest, the organization of the experimental protocols requires specific implementation details. For example, it is common place in behavioral experiments to familiarize participants with the materials (e.g. pictures) before the experiment proper or to present the target materials multiple times within and/or across conditions. To the extent that these "convenience manipulations" do not interact strongly with the effect of interest (for familiarization, see Alario et al., 2004; for repetition, see Figure 10 in Levelt et al. 1999; for both, see Navarrete, Mahon, \& Caramazza, 2010) they are typically not considered in detail. Yet, 
manipulations such as familiarization and repetition have been investigated in their own right in neurophysiological research. They have been shown to have substantial influences on brain activity, and on the processes engaged to perform a given task (Dhond, Buckner, Dale, Marinkovic, \& Halgren, 2001; Marinkovic et al., 2003; McDonald et al., 2010). Given that neurophysiological research on language production is increasingly guided by psycholinguistic behavioral protocols. It is crucial to test whether and how parameters that may be deemed secondary in behavioral studies, such as familiarization and repetition modulate the neural networks underlying performance in these protocols.

Here we address this issue based on two implementations of the picture naming task that are amongst the most popular in language production research. We will refer to those as the "sequential "and "blocked cyclic" picture naming protocols. The first protocol involves naming a sequence of pictures belonging to multiple semantic categories. Typically, these pictures are presented for naming only once, and participants are not familiarized with them (Howard, Nickels, Coltheart, \& Cole-Virtue, 2006). The second protocol involves naming repeatedly, within a block, a few pictures after participants have been familiarized with them (e.g., Damian, Vigliocco, \& Levelt, 2001; Roelofs, 2006).

\section{Research based on the sequential and blocked naming protocols}

Up to now, the sequential and blocked naming protocols have been used quite interchangeably to investigate processing stages involved in word production. For example, semantic interference effects are thought to reflect lexical selection processes. When words are produced in response to sequentially presented objects or definitions, performance decreases with the number of previously named items from the same semantic category (Howard et al., 2006). In the blocked protocol, performance also decreases if the items within a block belong to same category, compared to when they are semantically heterogeneous (Damian \& Als, 2005; Damian et al., 2001; McCarthy \& Kartsounis, 2000; note that performance increases when the items are phonologically rather than semantically related: Roelofs, 2006).

These two manifestations of semantic interference are most often construed as instances of the same phenomenon. That is, an increase in the duration or difficulty of semantically driven lexical retrieval, within the same processing pathway leading from object recognition to word articulation. Most notably, Oppenheim, Dell, \& Schwartz (2010) explicitly hypothesize the semantic interference effect stems from the same processing mechanisms in these two protocols. They more tentatively suggest that semantic interference could be tied to a single underlying cortical network involving the left inferior frontal gyrus (LIFG) and/or the left temporal lobe. This hypothesis has been recently challenged. Most notably, Belke \& Stielow (2013) concluded a review of the behavioral evidence by noting important differences between these two protocols. In particular, they highlight differences in the relative involvement of top-down processes across blocked and sequential naming.

Most of the empirical work discussed above on the sequential and blocked naming paradigms has relied on behavioral measures (in healthy and impaired speakers). Few studies have explored the neurophysiological signature of these protocols and effects. Costa, Strijkers, Martin, \& Thierry (2009) measured the event related potentials (ERP) in the 
sequential naming protocol, and their modulation by semantic context. They reported an amplitude modulation of the ERPs on posterior electrodes bilaterally, which was correlated to the magnitude of behavioral semantic interference during a time window between 200 and $400 \mathrm{~ms}$ approximately. We are not aware of other imaging or neurophysiological published studies of this protocol. There are more studies that have tested the blocked naming protocol. Using cortical source reconstruction from magneto-encephalographical (MEG) data, Maess, Friederici, Damian, Meyer, \& Levelt (2002) reported that semantic interference was associated with the left temporal region during a time window similar to that mentioned above. This is also reported by the ERP study of Aristei, Melinger, \& Abdel Rahman (2011), although the responses they observed were bilateral. Also using ERPs, Janssen, Carreiras, \& Barber (2011) reported that semantic interference was associated with an amplitude modulation, mostly observed in anterior electrodes, occurring between 220 and $450 \mathrm{~ms}$. These authors link this effect to input processes (e.g. visual and semantic identification of the picture) rather than word retrieval per se. Schnur et al. (2009) and Schnur, Schwartz, Brecher, \& Hodgson (2006) tested the same protocol in fMRI with a group of Broca's aphasics, as well as healthy speakers. Their results link semantic interference and phonological facilitation effects with temporal regions, but only semantic interference was associated with the LIFG. The authors highlighted the involvement of the LIFG in solving the conflict between semantically-related candidate words. Finally, an fMRI study conducted by Hocking, McMahon, \& de Zubicaray (2008) showed a greater activation in the left middle to posterior superior temporal gyrus as well as in the hippocampus, bilaterally, for the homogeneous than for the heterogeneous context.

Together, the studies of the blocked naming protocol broadly reveal a fronto-temporal network. This network is closely related to the standard network of picture naming (Damasio et al., 2004; Indefrey, 2011; Price, 2012), thought to be engaged in the sequential protocol. This could suggest that, across the sequential and the blocked naming protocols, the same cortical network is similarly engaged in naming processes (e.g., lexical selection) as has been implicitly or explicitly assumed in previous research (with the notable exception of Belke \& Stielow, 2013). However, the data do not provide a definite test of this hypothesis, particularly because the two protocols have never been directly compared with imaging or neurophysiological methods.

\section{The current study}

The goal of the current study was to test the hypothesis that the naming protocols under discussion reflect the same processes with the same time course. The alternative is that these two tasks rely, at least in part, on different processes and hence produce distinguishable electro-physiological signatures. To evaluate this alternative, we implemented and tested standard versions of the sequential and blocked naming protocols with a single pool of native speakers, all within the same experimental session. The protocols differ in terms of the familiarization and repetition of the stimuli (see below), which could significantly modulate the cortical network activity. In addition, each protocol included the usual semantic manipulation, which was expected to yield the semantic interference effect thought to reflect the core stage of word selection. We concurrently collected the speakers' EEG 
activity and vocal responses. The spatio-temporal signatures of the two protocols were compared on the basis of ERPs and source localization.

By testing the protocols as they have been designed in previous research, which was our goal, the direct protocol comparison inevitably conflated a contrast in familiarization (unfamiliar vs. familiar items in sequential vs. blocked naming, respectively) with a contrast in the number of item repetitions (no vs. many repetitions in sequential vs. blocked naming, respectively). This was explicitly considered in our data analysis. We always compared the effects of protocol with a "pure" effect of repetition computed within the blocked naming protocol. If the protocol effect was also seen in the (within-protocol) "pure" repetition contrast, the effect was attributed to repetition. If, however, different patterns were observed, then the protocol effect of interest was attributed to the familiarization procedure (see below for further details).

\section{Materials and Methods}

\subsection{Subjects}

ERPs were recorded in 22 right-handed healthy adults (mean age $=25.6 \pm 7.7$ years, 10 men). All were native speakers of French and had around 18 years of education. Informed consent was obtained in accordance with the University Hospital of Marseille institutional review board recommendations. Two participants were rejected from the electrophysiological analysis due to a low signal-to-noise ratio.

\subsection{Experimental Procedure}

For all the experiments, 108 object names were chosen from eighteen different semantic categories (six words per category; details in Appendix A). These words were matched in lexical frequency and number of syllables across categories. Simple object drawings corresponding to these words were selected from published picture databases (Alario \& Ferrand, 1999; Bonin, Peereman, Malardier, Meot, \& Chalard, 2003) or designed by us for the experiment. The pictures were black and white drawings in bitmap format $(245 \times 240$ pixels).

Two picture naming tasks were presented in succession. The first one, referred to as the sequential naming protocol, consisted in naming once the 108 pictures presented one after the other. The pictures were presented in three runs containing 36 items each ( 6 pictures from 6 semantic categories). In the original report of the cumulative semantic interference effect (Howard et al., 2006), the distance in trials between members of a given semantic category was carefully manipulated. Because this factor was not shown to influence the data, and because cumulative semantic interference has also been shown with a fixed lag (Runnqvist, Strijkers, Alario, \& Costa, 2012), we did not manipulate lag in our design ${ }^{1}$. This being said, the pictures within each run were arranged pseudo-randomly (Van Casteren \& Davis, 2006), thus creating two different lists with the constraints that adjacent trials did not involve items from the same semantic category nor items beginning with the same phoneme.

\footnotetext{
$1_{\text {The mean lag was }} 5.8$ (standard deviation: 2.97; range: 1-17).
} 
Besides its intrinsic interest, this first protocol served as a training phase and familiarization of material for the second protocol, namely the blocked naming protocol, in which 36 previously named pictures were used.

In this protocol, the pictures were named in blocks of six items, either from the same semantic category (semantically homogeneous blocks) or six different semantic categories (semantically heterogeneous blocks). The items were repeated five times within a block, yielding 30 trials per block. The order of the items within block was pseudo-randomized with the constraints that adjacent trials did not involve the same single item nor items beginning with the same phoneme. There were 6 homogeneous and 6 heterogeneous blocks, yielding 360 trials in total. We created 4 different block lists by pseudo-randomly arranging the order of the blocks to vary the alternation between homogeneous and heterogeneous contexts, again using the software MIX.

The experiment was controlled by E-Prime v2.0.1 (Psychology Software Tools, Pittsburgh, USA). The pictures were presented on the center of the screen within an angular size of $6^{\circ} \times$ $6^{\circ}$. Naming latencies were recorded with a microphone (audio Technica ATR20) placed 13 $\mathrm{cm}$ in front of the participant. Response times (in $\mathrm{ms}$ ) were automatically recorded by the software voice-key. A trial consisted of a fixation point (variable duration across trials, between 1400 and $2100 \mathrm{~ms}$ ), followed by the black and white target picture (presented for $1000 \mathrm{~ms})$.

Participants were seated in a sound- and light-attenuated room that was electrically shielded; facing a display monitor located $70 \mathrm{~cm}$ away in front of the subject. An experimenter was present in the soundproof room, sitting behind the participant; to monitor the performance and take written note of erroneous responses (error types described below in the results section). Participants were instructed to name the pictures as fast as possible while avoiding errors; they were asked to remain silent if they did not recognize the depicted object. The duration was approximately ten minutes for the sequential naming protocol, and approximately twenty minutes for the blocked naming protocol.

\subsection{EEG Recordings and Data Processing}

EEG was recorded using BrainAmp amplifiers and the BrainRecorder software (Brain Products GmbH, Munich, Germany) from an EEG cap (Quick-Cap, Compumedics Neuroscan, Charlotte, USA) with 60 scalp electrodes placed according to the International 10-10 system and referenced to the right mastoid (bandwith $=0.1-200 \mathrm{~Hz}$, sampling rate $=$ $1000 \mathrm{~Hz}$ ). Horizontal EOG was recorded from electrodes at the outer canthi of both eyes; vertical EOG was recorded from electrodes above and below the right eye. Scalp electrode impedances were kept below $5 \mathrm{k} \Omega$.

Off-line processing of EEG data was performed with the BrainVision Analyzer® software (Brain Products $\mathrm{GmbH}$, Munich, Germany). Continuous EEG data were re-referenced to an average reference and filtered digitally $(0.5-40 \mathrm{~Hz}, 12 \mathrm{~dB} / \mathrm{oct})$. As our time analysis window did not exceed $2 \mathrm{sec}$, we were not interested on slow activity (Delta range) whose periodic cycle is $<0.5 \mathrm{~Hz}$. Eye blinks were isolated and removed using an independent component analysis (Fast ICA algorithm). For 13 participants out of the 20 participants, motor artifacts 
were not removed efficiently so a second ICA decomposition was used (Jung et al., 2000). Epochs were created beginning $1000 \mathrm{~ms}$ before stimulus onset and lasting until $2000 \mathrm{~ms}$ post-stimulus. Only the epochs corresponding to correct behavioral responses were kept for the analysis (see section 2.5 for description of erroneous responses). In epochs containing artifacts, the artefactual channels were individually removed based on a semi-automatic threshold of potential values $( \pm 80 \mu \mathrm{V})$; this preserved the other channels of the epochs.

A baseline correction was applied between $-500 \mathrm{~ms}$ and $-100 \mathrm{~ms}$. Grand-average ERPs were computed for 10 different conditions. Two of these conditions concerned the effect of protocol: all trials in the sequential naming protocol, all trials in the blocked naming protocol (see below for details on the sub-sampling procedures we used to equate number of trials across conditions in this and other statistical comparisons). Two other conditions concerned the sequential naming protocol: the first two items of each semantic category and the last two items of each semantic category. Their comparison allows testing, within this protocol, of the semantic effect driven by the ordinal position within the semantic categories (Costa et al., 2009; Howard et al., 2006). The six other conditions concerned the blocked naming protocol. These were: trials from homogeneous blocks, the first two repetitions of pictures in homogeneous context, the last two repetitions of pictures in homogeneous context and exactly the same three conditions taken from heterogeneous blocks. These conditions allowed testing, within this protocol, the effect of semantic homogeneity and the effect of item repetition.

\subsection{Source localization}

sLORETA (standardized low resolution brain electromagnetic tomography) was used to estimate, for each condition, the sources of the ERP components shown to differ significantly at the sensor level (Pascual-Marqui, 2002; see http://www.uzh.ch/keyinst/ loreta.htm for details). sLORETA images represent the standardized electric activity at each voxel in neuroanatomic Montreal Neurological Institute (MNI) space as the exact magnitude of the estimated current density. Voxels that were unambiguously labeled as cortical grey matter, and that fell unambiguously within the brain compartment, were retained. This produced 6239 cortical grey matter voxels at $5 \mathrm{~mm}$ resolution. Anatomical labels as Brodmann areas are also reported using MNI space, with correction to Talairach space (Brett, Anton, Valabregue, \& Poline, 2002). Before applying sLORETA software, a signalto-noise ratio for each condition is computed in order to minimize false source localization due to noisy signals. The source localization was computed for each protocol (sequential and blocked naming) separately, first on the grand-average and then on each participant.

\subsection{Statistical Analysis}

Behavioral data-Following standard practice in behavioral studies of picture naming, the analysis of naming latencies did not include trials with responses faster than $300 \mathrm{~ms}$ (noisy anticipations or recording errors), naming errors (semantic paraphasias, hesitations, etc.), and omissions (no response). The complementary analysis of error rates was conducted by combining the two later types of trials. Analyses of variance (ANOVA) were first computed to contrast the sequential and blocked naming protocols (one-way repeatedmeasure ANOVA). We then performed ANOVAs on the naming latencies and the error 
rates to estimate the effect of semantic interference within each protocol. More specifically, a one-way repeated-measure ANOVA was performed on the sequential naming data to test for the effect of ordinal position within the semantic categories. A two-way repeatedmeasures ANOVA was conducted on the blocked naming protocol to estimate the effect of block type (i.e. heterogeneous versus homogeneous), the effect of item repetition, and their interaction.

Electrophysiological data-The EEG statistical analysis was conducted on the epoched single trial monopolar recordings from all participants for each condition. The analysis was performed with the EEGlab toolbox (Delorme \& Makeig, 2004).

To characterize the differences within and between protocols, we performed running Student t-tests. Because the data-sets compared differed in size (due to number of observations and/or number of errors), subsets of data with equal number of trials were compared in the following manner. For each comparison, we extracted from each data-set a number of trials equal to $80 \%$ of trials on the smaller data-set with a random uniform probability, and performed the statistical comparison on this sub-sample. We then iterated the sub-sampling operation 50 times. Periods of time in which at least 50 consecutive milliseconds showed significant differences between conditions in at least $80 \%$ of the extracted sub-samples were considered to reflect robust effects. The alpha level was originally set at .001 . When no differences were apparent at that level a more liberal threshold (.01 or .05) was explored, and significant differences at that level, if any, are reported. To visualize the direction of the differences across channels, we computed the sign of the difference between the absolute value of the electro-physiological signal from both conditions. This difference is represented on the statistical maps in the figures

We first compared the difference between the two picture naming protocols (sequential vs. blocked naming). Secondly, we tested the significance of the repetition effect within the blocked naming by contrasting trials from the first two and the last two occurrences. Finally, we tested for the presence of significant effects of semantic interference within each protocol: ordinal position effect for the sequential protocol and block type for the blocked naming protocol.

The cortical sources estimated with sLoreta on the grand average of each naming protocol were visually compared. Then, the sources estimated for each participant in each protocol were used to perform voxel-by-voxel between-group comparisons. Specifically, nonparametric statistical analyses of functional sLORETA images (Statistical nonParametric Mapping: SnPM; Nichols \& Holmes (2002) were performed employing a log-Fratio statistic for paired groups. The results correspond to maps of log-F-ratio statistics for each voxel, corrected for multiple testing (for further details, see http://www.uzh.ch/keyinst/ loreta.htm). Our analysis focused on the time-windows of the two early components revealed in the sensor-level statistical analysis. 


\section{Results}

\subsection{Comparison between the two naming protocols}

Behavioral results-Mean naming latencies were significantly slower in the sequential than in the blocked naming protocol $(819 \mathrm{~ms}$ vs. $675 \mathrm{~ms} ;(\mathrm{F}[1,21]=54, \mathrm{p}<.001))$. This difference in response timings will be considered in some detail when analyzing electrophysiological results in the next section. There were $8.4 \%$ errors in the sequential naming protocol (200 errors out of 2376 trials), and $2.8 \%$ in the blocked naming protocol (224 errors out of 7920 trials). This difference was significant $(F[1,21]=40.86, p<.001)$.

Electrophysiological results-The comparison of time course EEG activity elicited by picture naming in both protocols revealed similar early components (N1/P2) followed by a strong difference in the amplitude distribution from $200 \mathrm{~ms}$ onwards (Fig 1. A). Two major phenomena could be distinguished. First, in frontal electrodes, the N250/N400 complex was much larger in the sequential than in the blocked naming protocol. The time window showing significant differences spanned approximately from 200 to $400 \mathrm{~ms}$ (Fig 1. B). Second, in parietal and to some extent central electrodes, a positive component which was larger in the blocked than in sequential naming protocol started around $300 \mathrm{~ms}$ and peaked around 500. This component reached its maximum earlier, and was resolved faster, in the blocked naming protocol. The time window showing this significant difference spanned from 300 to $700 \mathrm{~ms}$, with blocked naming showing a stronger response between 300 to 600 $\mathrm{ms}$, and then, after its resolution, the sequences showing stronger (i.e. more sustained) activity until around $800 \mathrm{~ms}$.

The electrophysiological differences between protocols we just described could be related to the difference of average response speeds in the two tasks. In particular, but not only, the component peaking around $500 \mathrm{~ms}$ was stronger and was resolved faster in the protocol with the faster responses (blocks). To clarify this issue, a subsidiary analysis was performed in which the response times were equated across the two protocols. We sub-sampled the data by selecting pairs of trials with similar response times $( \pm 10 \mathrm{~ms})$ across protocols within every participant. This was possible because the distributions of naming latencies are always quite broad. We selected as many pairs of trials as was possible for every participant, and ended up with a total of 1092 pairs of trials in each protocol. The mean latency for the sequential naming protocol was $713 \mathrm{~ms}$ and $711 \mathrm{~ms}$ for the blocked naming protocol. The EEG data of this sub-sample was then analyzed as the full data set above. The electrophysiological pattern for each protocol and their significant difference were essentially identical to that observed with the whole data set (compare Figure 1 and Figure S1). This provides a strong argument against the view that speed of processing is responsible for the contrasts across protocols.

Source localizations-The sLORETA solution computed on the grand averages of each protocol revealed significant sources that were relatively similar sources across protocols for the early (presumably visual) component (around $100 \mathrm{~ms}$ ). Later, a component peaking around 220-230 ms involved ventral occipito-temporal sources, predominantly rightlateralized, as well as activation of posterior regions (Fig 2, top). A marginal difference ( $\mathrm{p}=$. 
08 corrected for multiple comparisons) was present between the source localizations observed in both protocols, as shown on the statistical map of differences (Fig 2, bottom left). The ventral stream is activated somewhat more strongly in the sequential protocol than in blocked protocol (red activation); on the other hand, the blocked protocol involved parieto-occipital regions much more (blue activation). In the $400 \mathrm{~ms}$ time window, a region encompassing the left inferior frontal gyrus (LIFG) was clearly activated in the sequential naming protocol while the temporo-parieto-occipital regions were still activated in the blocked naming protocol. The difference in localization across tasks was again a marginally significant ( $\mathrm{p}=0.07$, Fig 2 right). For the late component, it was not possible to find a reliable localization for either protocol. The sources revealed for this latency range were presumably too variable due to inter-trial jitter, which is expected to increase along the duration of the trial, (e.g., Maillard et al., 2011).

In summary, the sLORETA source reconstruction algorithm revealed meaningful and significant networks independently for each of the two protocols up to around $400 \mathrm{~ms}$. The contrasts between sources were only marginal $(.05<\mathrm{p}<.1)$, even at the time windows where significant differences were observed in the surface data.

\subsection{Item repetition manipulations within the blocked naming protocol}

The localization procedure suggests that different brain networks may be involved in the two protocols. Before interpreting these observations any further, we have to take into consideration that the two protocols were administrated always in the same order, and that they involved different amounts of item repetition. The next step of the analysis was intended to estimate how repetitions modulate the behavioral and electrophysiological responses, using the blocked naming data.

Behavioral results-A significant repetition effect was observed on naming latencies ( $\mathrm{F}$ $[4,84]=3.53, \mathrm{p}<.05)$, which interacted with semantic context $(\mathrm{F}[4,84]=3.27, \mathrm{p}<.05)$ (see Fig S2 in supplementary materials). A significant repetition effect was also observed on the error rates $(\mathrm{F}[4,84]=5.96, \mathrm{p}<.001)$.

Electrophysiological results-We contrasted the first two and last repetitions across blocks. The comparison of the time course of activation for these two conditions showed a significant difference in the 500-600 time window only. This P600 component was larger for the first than for the last two repetitions, irrespective of the homogeneity condition (Fig 3. A). This difference is observed at centro-parietal recording sites bilaterally (Fig 3.B).

\subsection{Semantic manipulation within the sequential naming protocol (ordinal position)}

We finally consider the semantic manipulations thought to target the specific stage of lexicosemantic retrieval, first within the sequential protocol (this section) and then within the blocked protocol (next section).

Behavioral results - The ANOVAs on naming latencies averaged per ordinal position revealed a significant effect of ordinal position on naming latencies $(F[5,21]=11, p<.001)$, and a significant effect on error rates $(F[1,21]=1.07, \mathrm{p}<.05$; see Fig $\mathrm{S} 2$ in supplementary 
material). Because the behavioral difference was maximal between the first two (771ms) and the last two ordinal positions (839 ms) (subsidiary ANOVA: F (3, 21) =13.99, p<.001; Fig 4. A), we focused the EEG analysis on these conditions.

Electrophysiological results-Figure 4.B presents the time course of scalp ERP elicited by the sequential naming protocol, shown over four bilateral regions of interest, illustrated by one representative electrode each.

The semantic contrast (comparison between the first two and last two ordinal positions within each semantic category) did not show any significant difference along the time course of ERPs, neither in amplitude nor in latency. This absence of effect stands in contrast with the significant effect of ordinal position on EEG amplitude reported in Costa et al. (2009). For the sake of comparison, we analyzed our data with the method used by those authors, namely correlations between response times and ERP amplitude. No significant effect was seen there either (see Fig. S3 in supplementary materials).

\subsection{Semantic homogeneity within blocked naming protocol}

Behavioral results-The ANOVA revealed a significant difference of performance between the homogeneous and the heterogeneous contexts (691 ms vs. $658 \mathrm{~ms}$; F [1,21]= $49.54, \mathrm{p}<.001)$ in the naming latencies, and a marginal difference in the error rate $(3.28 \%$ vs. 2.37\%; F[1,21]= 4, $\mathrm{p}=.06$ ) (see Fig 5. A). As expected, participants were slower, and tended to make more errors, in the homogeneous than in the heterogeneous blocks.

Electrophysiological results-No significant difference in the time course of ERPs was observed between the semantically homogeneous versus heterogeneous contexts (Fig 5 B.).

\section{Discussion}

Our goal was to investigate the spatio-temporal dynamics of visual object naming processes. Two variants of the picture naming task were implemented and tested within participants: the sequential and the blocked naming protocols. These two variants are frequently used to investigate word production and as such are considered, implicitly or explicitly, to rely on the same underlying processes. In addition, we estimated the effect of semantic context in both protocols.

\section{Summary of findings}

The behavioral effects we observed were as expected. In contrast, the neural responses were more revealing. Despite general similarity in the components elicited by the sequential and the blocked naming protocols, a direct comparison of their time courses revealed significant modulations of amplitude of the components, as early as $200 \mathrm{~ms}$. This observation is quite unexpected in the context of current theoretical thinking, and practical use, of the two protocols (although see Belke \& Stielow, 2013). Importantly, these electrophysiological differences cannot be explained by a shift or modulations of the components resulting from different response speeds. Finally, the semantic manipulation did not yield any significant electrophysiological effect. 


\section{Expected pattern in the behavioral data}

Naming latencies were significantly slower and the number of errors was higher in sequential naming than in blocked naming. This was expected, given the purposed organization of the testing session. Following common practice, the sequential naming protocol was delivered first, without any prior familiarization with the materials. Following common practice too, the blocked naming protocol was then performed using items that had been named in the previous run. In earlier reports of the blocked naming protocol, participants always named the materials at least once before the experiment proper (to our knowledge the performance in such familiarization run has never been reported, as is done here). Note finally that the blocked naming protocol involves repetitions of each item per block (in our design, five).

The behavioral results show significant semantic interference effects in both protocols. These were similar in magnitude to those reported in previous studies. In blocked naming, the effect was similar to that described in previous reports (e.g. Damian et al., 2001). In addition, when the data were analyzed without the first repetition, following Belke \& Stielow's study (2013), the semantic context effect was still significant, but there was no significant increase or decrease of its size with repetition (i.e. no significant interaction; $p=$. 57). This suggests that, in our blocked naming data at least, semantic interference was not cumulative. In sequential naming, the semantic interference did not show a strictly linear relationship with ordinal position in the category (Costa et al., 2009; Howard et al., 2006) but rather a marked attenuation of cumulative interference for the fifth and sixth ordinal positions. The exact reasons for this attenuation remain unclear. Note that the cited studies used five items per category, but that the effect has been shown to span at least ten items per category (Experiment 1 in Runnqvist et al., 2012). In any event, because there was a large and significant difference in response times between the first two and the last two ordinal positions, and thus we focused on this comparison for the analysis of the electrophysiological data. This choice was also made because such binary semantic was directly comparable with semantic contrast in the blocked naming protocol.

In short, as was expected, the behavioral results show speedy and efficient naming, as well as significant semantic interference effects in both protocols. The protocols were thus successful in eliciting the cognitive processes of interest.

\section{Parameters that may underlie the electrophysiological contrast across protocols}

Significant differences between protocols were present from $200 \mathrm{~ms}$ onwards, visible over three ERP components that peaked around the latencies $250 \mathrm{~ms}, 400 \mathrm{~ms}$, and $600 \mathrm{~ms}$. To interpret these differences, the first possibility to consider is that protocol differences are driven by item repetition. Sequence naming involved, in essence, the first repetition of items that would later be repeated again multiple times in the blocked naming protocol ${ }^{2}$. To test this hypothesis, we sought to isolate the neurophysiological signature of repetition from that of the protocol contrast. This was achieved by comparing the early and late repetitions

\footnotetext{
${ }^{2}$ Recall that we implemented the two protocols with their standard procedures. Sequential naming was always performed first, and involved novel items without any repetition. Blocked naming was then performed on items that had been familiarized during sequential naming, and involved item repetitions.
} 
within the blocked naming protocol. There were no repetition effects within the block protocol up until around $500 \mathrm{~ms}$ where the amplitude was larger in the first than in the last two repetitions (Figure 3), presumably reflecting a habituation effect (Gruber \& Müller, 2005). Notably, this effect is opposite to that found when comparing the protocols with one another, where sequential naming elicited a smaller component than blocked naming during this same time window. Thus, protocol differences do not merely reflect a habituation effect driven by item repetition or, at least, the first unfamiliar ("never-seen/said-before") presentation has a different status from the following repetitions,

The early visual perceptive components P1/N2 were never affected by the protocol contrast. This absence of difference suggests that the contrasts across protocols occurred after sensory processes. This may not be surprising, as the modulation of these components by familiarity or repetition only occurs under specific circumstances (Grill-Spector, Henson, \& Martin, 2006).

An alternative possibility is that the ERP modulations across protocols are directly linked to the variation in response speeds (on average, a $150 \mathrm{~ms}$ difference). ERPs elicited by slower, more variable, responses may be shifted in time. Also, they may last longer and have reduced amplitude. Our analysis involving pairs of protocol trials matched on their naming latencies showed that this explanation does not account for the data. Remarkably, the contrast between protocols was essentially the same for equated response times. In addition, the significant difference between protocols is not a homogeneous modulation of amplitude over the time course of response preparation. Early on, $200 \mathrm{~ms}$ onwards, the sequential naming protocol yielded significantly stronger responses over frontal electrodes, especially on the left. Later on, $300 \mathrm{~ms}$ onwards, the blocked naming protocol yielded significantly stronger responses over posterior electrodes, especially on the left.

Altogether these observations suggest that the protocol contrast reflects, from $200 \mathrm{~ms}$ onwards, a change in the underlying neural and presumably cognitive processes, rather than the modulation of a network that would be stable across protocols. The changes in processing are likely driven by the familiarization occurring when the items are first seen/ said. This interpretation argues against the view that a single mechanism operates similarly in both naming protocols (Oppenheim et al., 2010), but is consistent with the general idea of differential processes across protocols (Belke \& Stielow, 2013).

\section{Interptreting protocol contrasts within word production models}

Indefrey (2011) has synthesized much of the current research in his influential model of word production. The model describes the cognitive stages and associated time-windows that may be engaged in a task such as picture naming. The time window around $200 \mathrm{~ms}$ is associated with lexical retrieval. The time window around $300 \mathrm{~ms}$ is associated with phonological processing and syllabification. Finally, the time window around $500 \mathrm{~ms}$ is associated with phonetic plans or with phonetic encoding. Interpreted within this model, the sustained differences we observed across protocols would reflect modulations of all these three processing stages. In addition to this core word-production interpretation, it is interesting to consider the ERP observed components in the broader context of studies involving related tasks. 
The "lexical component", peaking around $250 \mathrm{~ms}$, has also been related to the processing of high level visual representation of objects, a transitional stage between perceptual and conceptual representations (Maillard et al., 2011; Marinkovic et al., 2003; Schweinberger, Huddy, \& Burton, 2004). It is not unlikely that these two successive processes overlap in time (see also Hart et al., 1998). The component peaking around $400 \mathrm{~ms}$ is comparable to the results of various word or object recognition ERPs studies, in which a frontal activation starting around $400 \mathrm{~ms}$ post-stimulus has been linked to strategic memory retrieval (Wilding \& Rugg, 1996, 1997). While phonological processing presumably involves memory retrieval, the processes occurring during this time window have not been explored in much detail in this task (although see Laganaro, Python, \& Toepel, 2013, and related work). Strong interpretations of what information is being retrieved from memory during this timewindow might stilll be premature.

Finally, the positive component spanning between 350 and $750 \mathrm{~ms}$ is quite comparable to the P600 component, which is associated with the detection of syntactic or semantic anomalies (Van Herten, Kolk, \& Chwilla, 2005), as well as with episodic memory processes. Regarding the latter, when participants are asked to judge whether words or pictures have occurred in a prior study list, items recognized as old elicit more positive ERPs than new items correctly rejected. This old/new effect thus reflects successful retrieval and, for words, it is observed in a large latency range of roughly $400-800 \mathrm{~ms}$ after stimulus onset (Yonelinas, 2002; for review). Importantly, the reliable old/new effect can also occur incidentally, when participants are not explicitly asked to make memory judgments but some items may be spontaneously recognized in the course of another task (Van Petten \& Senkfor, 1996). In this context, the P600-like component we recorded may not reflect syllabification and articulation but could (also) correspond to the incidental building and retrieval of a memory trace, which combines the representation of the item that is being identified and the associated word. Consistent with this general interpretation, this component was more positive, earlier and sharper following familiarization (i.e. in the blocked protocol) than before it (i.e. in the sequential naming protocol). In addition, the component was attenuated across repetitions in the blocked naming protocol (Düzel, Yonelinas, Mangun, Heinze, \& Tulving, 1997; Van Petten \& Senkfor, 1996).

In short, the early ERP components apparent in our data can be tentatively interpreted within Indefrey's (2011) model of word production, but alternative interpretations cannot be excluded. The later ERP component, on the other hand, has no clear relationship with response speed, which makes it unlikely that it (only) reflects a pre-articulation tied to response triggering such as phonetic processing (see also Riès et al., 2013 for discussion of response-locked components). This component seems to reflect incidental processes sensitive to the familiarity of the materials.

\section{Interpretation of sources localized with the sLORETA algorithm}

Neural sources were identified with sLORETA for each protocol independently, We discuss those briefly while keeping in mind the limitations of localizing brain activity on the basis of EEG data, The two protocols engaged similar neural regions until about $250 \mathrm{~ms}$. From there onwards, sequential naming elicited a left frontal activation (in the vicinity of the left 
inferior frontal gyrus, LIFG; Figure 2) which lasted until around $700 \mathrm{~ms}$ post-stimulus. In contrast, in blocked naming the posterior regions (temporo-parieto-occipital areas) were the most active. These differences are suggestive of the engagement of different cortical networks in the two protocols. Sequence naming recruited left frontal regions, especially the LIFG, for which a large body of evidence has shown that these left frontal regions support the selection and retrieval of lexico-semantic information (Ashby, Sanders, \& Kingston, 2009; Wheat, Cornelissen, Frost, \& Hansen, 2010; Yvert, Perrone-Bertolotti, Baciu, \& David, 2012). Blocked naming recruited posterior regions such as the left temporo-parietal region, which has been associated with the phonological buffer in working memory models (Baddeley, 2003) or, in (related) processing terms, to the binding of phonological input and output codes (Hickok \& Poeppel, 2007; Hickok, 2012). The recruitment of phonological working memory is plausible in the context of blocked naming given its reliance on the repeated naming of a relatively small set of items (see also Norton \& Wolf, 2012).

Importantly, however, while the localization performed within each protocol provides a reliable description ("zero-error"; Pascual-Marqui, 2002) of regions engaged in each protocol, the differences between protocols were only marginally significant across participants $(.05<\mathrm{p}<.1)$. Therefore, the interpretation of the protocol contrast in terms of specific neural sources and their associated processes is only suggestive at this time.

\section{Absence of semantic interference in the electrophysiological signal}

The behavioral semantic interference effects we observed are thought to index increased semantico-lexical processing during word selection. There was no trace of these effects in the electrophysiological data. None of the components showed any significant amplitude modulation as a function of the semantic manipulations, be it in sequential or blocked naming. This absence of semantic modulation is in contrast with a few previous reports (Aristei et al., 2011; Costa et al., 2009; Janssen et al., 2011; Maess et al., 2002).

We could not find a straightforward explanation for the discrepancy between our data and those from previous reports. In particular, there are no obviously causal differences in the experimental procedures across studies. First, there is no a priori reason that semantic effects should not surface in French, as they surface in English or Spanish (see Figure 1 in Costa, Alario, \& Sebastián-Gallés (2007), on the stability across languages of the semantic interference effect in the picture-word interference protocol). Our implementation of the sequential protocol did not have fillers, as was the case in Costa et al. (2009); yet these filler pictures appear exactly as target pictures in the stream of trials seen by participants, and they call for the same kind of naming response. Moreover, the supplementary analysis made with the same cluster of electrodes and with the statistical model used by Costa et al. (2009) did not show any significant effect of ordinal position on ERP amplitude (Figure S3). Our protocol involved similar number of items (108 vs. 120) from about the same number of semantic categories (18 vs. 24: note that the categories in Costa et al., 2009, originally from Howard et al., 2006, stem from very fine grained distinctions; see Alario \& Moscoso del Prado Martín, 2010). As for our implementation of the blocked naming protocol, it involved more items (36 vs. 25 ), more categories (6 vs. 5), and more participants (20 vs. 18) than were tested by Janssen et al. (2011). One difference between our protocol and that of 
Janssen et al. (2011) is that the familiarization phase used here is not absolutely canonical. When our participants started the blocked naming protocol, they had been familiarized not only with the 36 experimental items (as is usually done) but also with the remaining 72 items used in sequential but not blocked naming. Moreover, the pictures seen in the sequential (experimental) protocol before the blocked naming protocol already induced a persistent semantic manipulation. In the standard familiarization procedure preceding blocked naming, there is no (implicit) manipulation of semantics and, accordingly, no effects are reported. It is really not apparent how these minor differences may contribute to attenuate the semantic contrast in the electrophysiological data. Pending further evidence, the absence, for both protocols, of semantic effects in our electrophysiological data may signal that these effects are small in magnitude or highly susceptible to sources of variance that remain to be investigated beyond the few available studies (Aristei et al., 2011; Costa et al., 2009; Janssen et al., 2011; Maess et al., 2002).

\section{Conclusion}

We compared the electrophysiological signatures of two popular visual naming protocols that were matched in their input materials (pictured objects) and output responses (overt naming), and that are commonly thought to elicit similar cognitive processes. Contrasting the sequential and blocked naming protocols revealed characteristic and significantly distinct patterns of electrophysiological activities at the sensor level. These differences were not induced by differences in the naming latencies between protocols. There was a relatively minor effect of repetition, presumably due to habituation suppression, which modulated the latest electrophysiological responses. The broad differences across protocols are attributed to the familiarization induced by the first encounter with the materials. This effect was not a homogenous modulation of the intensity of the components, suggesting that the protocol contrast reflects a substantial change in the underlying neural and presumably cognitive activity. The source localization procedure tentatively suggests that frontal regions may be more recruited by the sequences and posterior regions more by the blocks. Unfortunately, the semantic interference effect was not present in our electrophysiological data, thus preventing stronger conclusions about cognitive loci. Overall, then, the picture naming process is substantially modulated by specific protocol requirements controlled by the familiarity and, to a much lesser degree, the repetition of materials

\section{Supplementary Material}

Refer to Web version on PubMed Central for supplementary material.

\section{Acknowledgments}

The authors acknowledge financial support from the European Research Council under the European Community's Seventh Framework Program (FP7/2007-2013 Grant agreement n 263575), a grant from CPER-Telius InsermInria, post-doctoral grants from the Fyssen foundation and the National Institute On Deafness And Other Communication Disorders of the National Institutes of Health under Award Number F32DC013245 awarded to S.R., as well as institutional support from the Brain and Language Research Institute and the Féderation de Recherche 3C (both at Aix-Marseille Université). We thank Patrick Marquis for help with participant testing, Mederic Descoins for help with EEG analysis, and Julien Krieg, Jean Michel Badier and Christian Benar for help with statistical analysis. 


\section{Appendix A}

Accessoires (Accessories) ${ }^{*}$ : bonnet (cap), casquette (cap), ceinture (belt), chapeau (hat), cravate (tie), masque (mask)

Animaux (Animals): chien (dog), cochon (pig), lapin (rabbit), mouton (sheep), souris (mouse), vache (cow)

Bâtiments (Buildings)* : chateau (castle), igloo (igloo), moulin (mill), niche (housedog), phare (lighthouse), tente (tent)

Ustensiles de cuisiene (Kitchen utensils) ${ }^{*}$ : assiette (plate), couteau (knife), cuillere (spoon), fourchette (fork), passoire (sieve), poele (stove)

Electroménager (Household Appliances): passoire (kettle), cafetiere (coffee pot), four (oven), gaziniere (cooker), ventilateur (fan)

Fruits ${ }^{*}$ : banane (banana), cerise (cherry), citron (lemon), fraise (strawberry), poire (pear), pomme (apple)

Légumes (Vegetables): asperge (asparagus), carotte (carrot), mais (but), oignon (onion), poivron (pepper), tomate (tomato)

Récipients (Containers): biberon (baby bottle), casserole (pan), seau (bucket), tasse (cup), theiere (teapot), vase (vase)

Parties d'habitat (Pieces of home): balcon (balcony), banc (bench), fenetre (window), porte (door), serrure (lock), toit (roof)

Meubles (Furniture) ${ }^{*}$ : baignoire (bath), bureau (office), canape (sofa), chaise (chair), etagere (shelf), lit (bed)

Multimedia: camera, clavier (keyboard), microphone, souris (mouse), telephone (phone), television

Instrument de musique (Musical Instruments) ${ }^{*}$ : batterie (battery), guitare (guitar), piano, saxophone, trompette (trumpet), violon (violin)

Outils (Tools): brouette (wheelbarrow), pelle (shovel), perceuse (drill), pince (pliers), rateau (rake), tondeuse (mower)

Papeterie (Stationery): calendrier (calendar), lampe (lamp), lettre (letter), livre (book), tampon (buffer), trousse (kit)

Salle de bain (Bathroom): brosse (brush), douche (shower), miroir (mirror), rasoir (shaver),robinet (tap), serviette (towel)

*semantic categories used in both protocols. 
Types de chaussures (Shoes): botte (boot), chaussure (shoe), palme (palm leaf), roller, sabot (clog), sandale (sandal)

Véhicule (Vehicle): avion (plane), bateau (boat), bus, camion (truck), moto (motorcycle), train

Vêtements (Clothing): chaussette (sock), chemise (shirt), jupe (skirt), pantalon (pants), robe (dress), tshirt

\section{References}

Alario, F.-Xavier; Ferrand, L. A set of 400 pictures standardized for French: Norms for name agreement, image agreement, familiarity, visual complexity, image variability, and age of acquisition. Behavior Research Methods. 1999; 31(3):531-552.

Alario, F.-Xavier; Ferrand, L.; Laganaro, M.; New, B.; Frauenfelder, U.; Segui, J. Predictors of picture naming speed. Behavior Research Methods. 2004; 36(1):140-155.

Alario, F.-Xavier; Moscoso del Prado Martín, F. M. del P. On the origin of the < < cumulative semantic inhibition > > effect. Memory \& Cognition. 2010; 38(1):57-66. [PubMed: 19966239]

Aristei S, Melinger A, Abdel Rahman R. Electrophysiological chronometry of semantic context effects in language production. Journal of cognitive neuroscience. 2011; 23(7):1567-1586. [PubMed: 20515409]

Ashby J, Sanders LD, Kingston J. Skilled readers begin processing sub-phonemic features by $80 \mathrm{~ms}$ during visual word recognition: Evidence from ERPs. Biological Psychology. 2009; 80(1):84-94. [PubMed: 18456383]

Baddeley A. Working memory: looking back and looking forward. Nature Reviews Neuroscience. 2003; 4(10):829-839.

Belke E, Stielow A. Cumulative and non-cumulative semantic interference in object naming: Evidence from blocked and continuous manipulations of semantic context. The Quarterly Journal of Experimental Psychology. 2013; 0(0):1-26.

Bock K. Language production: Methods and methodologies. Psychonomic Bulletin \& Review. 1996; 3(4):395-421. [PubMed: 24213975]

Bonin P, Peereman R, Malardier N, Meot A, Chalard M. A new set of 299 pictures for psycholinguistic studies: French norms for name agreement, image agreement, conceptual familiarity, visual complexity, image variability, age of acquisition, and naming latencies. Behavior Research Methods Instruments \& Computers. 2003; 35(1):158-167.

Brett M, Anton JL, Valabregue R, Poline JB. Region of interest analysis using an SPM toolbox. NeuroImage. 2002; 16(2):497.

Caramazza A, Hillis AE. Where do semantic errors come from? Cortex: A Journal Devoted to the Study of the Nervous System and Behavior. 1990; 26(1):95-122. [PubMed: 2354648]

Costa, A.; Alario, FX.; Sebastián-Gallés, N. Cross-linguistic research on language production. Handbook of psycholinguistics. Oxford University Press; Oxford, UK: 2007.

Costa A, Strijkers K, Martin C, Thierry G. The time course of word retrieval revealed by event-related brain potentials during overt speech. Proceedings of the National Academy of Sciences. 2009; 106(50):21442-21446.

Damasio H, Tranel D, Grabowski T, Adolphs R, Damasio A. Neural systems behind word and concept retrieval. Cognition. 2004; 92(1-2):179-229. 1. [PubMed: 15037130]

Damian MF, Als LC. Long-lasting semantic context effects in the spoken production of object names. Journal of Experimental Psychology: Learning, Memory, and Cognition. 2005; 31(6):1372.

Damian MF, Vigliocco G, Levelt WJM. Effects of semantic context in the naming of pictures and words. Cognition. 2001; 81(3):B77-B86. [PubMed: 11483172]

De Zubicaray GI, McMahon KL. Auditory context effects in picture naming investigated with eventrelated fMRI. Cognitive, Affective, \& Behavioral Neuroscience. 2009; 9(3):260-269. 
Dell GS, Burger LK, Svec WR. Language production and serial order: A functional analysis and a model. Psychological Review. 1997; 104(1):123-147. [PubMed: 9009882]

Delorme A, Makeig S. EEGLAB: an open source toolbox for analysis of single-trial EEG dynamics including independent component analysis. Journal of Neuroscience Methods. 2004; 134(1):9-21. [PubMed: 15102499]

Dhond RP, Buckner RL, Dale AM, Marinkovic K, Halgren E. Spatiotemporal maps of brain activity underlying word generation and their modification during repetition priming. The Journal of neuroscience: the official journal of the Society for Neuroscience. 2001; 21(10):3564-3571. [PubMed: 11331385]

Düzel E, Yonelinas AP, Mangun GR, Heinze H-J, Tulving E. Event-related brain potential correlates of two states of conscious awareness in memory. Proceedings of the National Academy of Sciences. 1997; 94(11):5973-5978.

Glaser WR. Picture naming. Cognition. 1992; 42(1-3):61-105.

Grill-Spector K, Henson R, Martin A. Repetition and the brain: neural models of stimulus-specific effects. Trends in Cognitive Sciences. 2006; 10(1):14-23. [PubMed: 16321563]

Gruber T, Müller MM. Oscillatory brain activity dissociates between associative stimulus content in a repetition priming task in the human EEG. Cerebral Cortex. 2005; 15(1):109-116. [PubMed: 15238442]

Hart J, Crone NE, Lesser RP, Sieracki J, Miglioretti DL, Hall C, Sherman D, Gordon B. Temporal dynamics of verbal object comprehension. Proceedings of the National Academy of Sciences. 1998; 95(11):6498-6503.

Hickok G. The cortical organization of speech processing: Feedback control and predictive coding the context of a dual-stream model. Journal of Communication Disorders. 2012; 45(6):393-402. [PubMed: 22766458]

Hickok G, Poeppel D. The cortical organization of speech processing. Nature Reviews Neuroscience. 2007; 8(5):393-402.

Hocking J, McMahon KL, De Zubicaray GI. Semantic Context and Visual Feature Effects in Object Naming: An fMRI Study using Arterial Spin Labeling. Journal of Cognitive Neuroscience. 2008; 21(8):1571-1583. [PubMed: 18823254]

Howard D, Nickels L, Coltheart M, Cole-Virtue J. Cumulative semantic inhibition in picture naming: Experimental and computational studies. Cognition. 2006; 100(3):464-482. [PubMed: 16413014]

Indefrey P. The spatial and temporal signatures of word production components: a critical update. Frontiers in psychology. 2011; 2:1-16. [PubMed: 21713130]

Indefrey P, Levelt WJM. The spatial and temporal signatures of word production components. Cognition. 2004; 92(1):101-144. [PubMed: 15037128]

Janssen N, Carreiras M, Barber HA. Electrophysiological effects of semantic context in picture and word naming. NeuroImage. 2011; 57(3):1243-1250. [PubMed: 21600993]

Jung T-P, Makeig S, Humphries C, Lee T-W, McKeown MJ, Iragui V, Sejnowski TJ. Removing electroencephalographic artifacts by blind source separation. Psychophysiology. 2000; 37(2):163178. [PubMed: 10731767]

Laganaro M, Python G, Toepel U. Dynamics of phonological-phonetic encoding in word production: Evidence from diverging ERPs between stroke patients and controls. Brain and Language. 2013; 126(2):123-132. [PubMed: 23707932]

Levelt, WJM. Speaking: From intention to articulation. MIT Press; Cambridge: MA: 1993. p. C

Levelt WJM, Roelofs A, Meyer AS. A theory of lexical access in speech production. Behavioral and Brain Sciences. 1999; 22(1):1-72. [PubMed: 11301520]

Levelt, Willem J.M. Accessing words in speech production: Stages, processes and representations. Cognition. 1992; 42(1-3):1-22. [PubMed: 1582153]

Llorens A, Trébuchon A, Liégeois-Chauvel C, Alario F-X. Intra-Cranial Recordings of Brain Activity During Language Production. Frontiers in Psychology. 2011; 2

Maess B, Friederici AD, Damian M, Meyer AS, Levelt WJM. Semantic category interference in overt picture naming: Sharpening current density localization by PCA. Journal of cognitive neuroscience. 2002; 14(3):455-462. [PubMed: 11970804] 
Maillard L, Barbeau EJ, Baumann C, Koessler L, Bénar C, Chauvel P, Liégeois-Chauvel C. From perception to recognition memory: time course and lateralization of neural substrates of word and abstract picture processing. Journal of Cognitive Neuroscience. 2011; 23(4):782-800. [PubMed: 20146594]

Marinkovic K, Dhond RP, Dale AM, Glessner M, Carr V, Halgren E. Spatiotemporal Dynamics of Modality-Specific and Supramodal Word Processing. Neuron. 2003; 38(3):487-497. [PubMed: 12741994]

McCarthy RA, Kartsounis LD. Wobbly words: Refractory anomia with preserved semantics. Neurocase. 2000; 6(6):487-497.

McDonald CR, Thesen T, Carlson C, Blumberg M, Girard HM, Trongnetrpunya A, Sherfey JS, Devinsky O, Kuzniecky R, Dolye WK, Cash SS, Leonard MK, Hagler DJ Jr. A. M. Halgren E. Multimodal imaging of repetition priming: Using fMRI, MEG, and intracranial EEG to reveal spatiotemporal profiles of word processing. NeuroImage. 2010; 53(2):707-717. [PubMed: 20620212]

Navarrete E, Mahon BZ, Caramazza A. The cumulative semantic cost does not reflect lexical selection by competition. Acta Psychologica. 2010; 134(3):279-289. [PubMed: 20347062]

Nichols TE, Holmes AP. Nonparametric permutation tests for functional neuroimaging: A primer with examples. Human Brain Mapping. 2002; 15(1):1-25. [PubMed: 11747097]

Nickels, L. Spoken word production.. In: Rapp, B., editor. A Handbook of Cognitive Neuropsychology. Psychology Press; Philadelphia: 2001. p. 291-320.

Norton ES, Wolf M. Rapid automatized naming (RAN) and reading fluency: Implications for understanding and treatment of reading disabilities. Annual review of psychology. 2012; 63:427452.

Oppenheim GM, Dell GS, Schwartz MF. The dark side of incremental learning: A model of cumulative semantic interference during lexical access in speech production. Cognition. 2010; 114(2):227-252. [PubMed: 19854436]

Pascual-Marqui RD. Standardized low-resolution brain electromagnetic tomography (sLORETA): technical details. Methods Find Exp Clin Pharmacol. 2002; 24(Suppl D):5-12. [PubMed: 12575463]

Price CJ. A review and synthesis of the first 20 years of PET and fMRI studies of heard speech, spoken language and reading. NeuroImage. 2012; 62(2):816-847. [PubMed: 22584224]

Rapp B, Goldrick M. Discreteness and interactivity in spoken word production. Psychological Review. 2000; 107(3):460-499. [PubMed: 10941277]

Riès S, Janssen N, Burle B, Alario F-X. Response-Locked Brain Dynamics of Word Production. PLoS ONE. 2013; 8(3):e58197. [PubMed: 23554876]

Roelofs A. The influence of spelling on phonological encoding in word reading, object naming, and word generation. Psychonomic bulletin \& review. 2006; 13(1):33-37. [PubMed: 16724765]

Runnqvist E, Strijkers K, Alario F-X, Costa A. Cumulative semantic interference is blind to language: Implications for models of bilingual speech production. Journal of Memory and Language. 2012; 66(4):850-869.

Schnur TT, Schwartz MF, Brecher A, Hodgson C. Semantic interference during blocked-cyclic naming: Evidence from aphasia. Journal of Memory and Language. 2006; 54(2):199-227.

Schnur TT, Schwartz MF, Kimberg DY, Hirshorn E, Coslett HB, Thompson-Schill SL. Localizing interference during naming: Convergent neuroimaging and neuropsychological evidence for the function of Broca's area. Proceedings of the National Academy of Sciences. 2009; 106(1):322327.

Schweinberger SR, Huddy V, Burton AM. N250r: a face-selective brain response to stimulus repetitions. Neuroreport. 2004; 15(9):1501. [PubMed: 15194883]

Van Casteren M, Davis MH. Mix, a program for pseudorandomization. Behavior research methods. 2006; 38(4):584-589. [PubMed: 17393828]

Van Herten M, Kolk HHJ, Chwilla DJ. An ERP study of P600 effects elicited by semantic anomalies. Cognitive Brain Research. 2005; 22(2):241-255. [PubMed: 15653297] 
Van Petten C, Senkfor AJ. Memory for words and novel visual patterns: Repetition, recognition, and encoding effects in the event-related brain potential. Psychophysiology. 1996; 33(5):491-506. [PubMed: 8854736]

Wheat KL, Cornelissen PL, Frost SJ, Hansen PC. During Visual Word Recognition, Phonology Is Accessed Within $100 \mathrm{Ms}$ and May Be Mediated by a Speech Production Code: Evidence from Magnetoencephalography. The Journal of Neuroscience. 2010; 30(15):5229-5233. [PubMed: 20392945]

Wilding EL, Rugg MD. An event-related potential study of recognition memory with and without retrieval of source. Brain. 1996; 119(3):889-905. [PubMed: 8673500]

Wilding EL, Rugg MD. An event-related potential study of memory for words spoken aloud or heard. Neuropsychologia. 1997; 35(9):1185-1195. [PubMed: 9364489]

Yonelinas AP. The Nature of Recollection and Familiarity: A Review of 30 Years of Research. Journal of Memory and Language. 2002; 46(3):441-517.

Yvert G, Perrone-Bertolotti M, Baciu M, David O. Dynamic Causal Modeling of Spatiotemporal Integration of Phonological and Semantic Processes: An Electroencephalographic Study. Journal of Neuroscience. 2012; 32(12):4297-4306. [PubMed: 22442091] 


\section{Highlights}

- We compared two naming protocols thought to recruit the same cognitive processes

- Semantic interference was observed in the behavior but not in the EEG data

- The two protocols elicited an intricate pattern of EEG activity differences

- Protocol differences were not due to response speed, but to item familiarity

- Picture naming processes can be modulated by specific protocol requirements 
A.

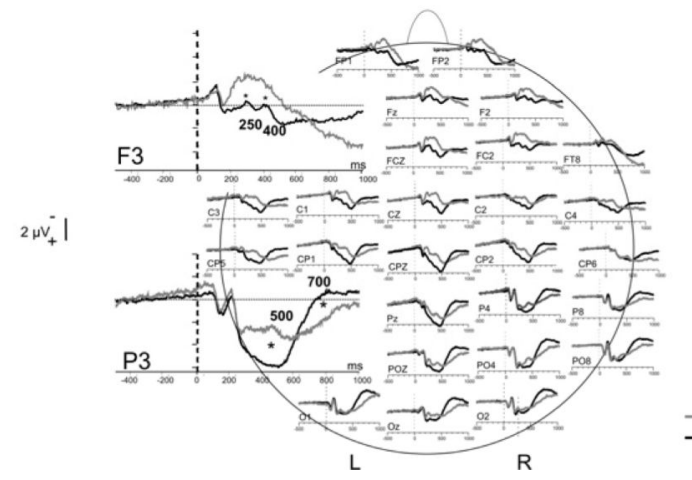

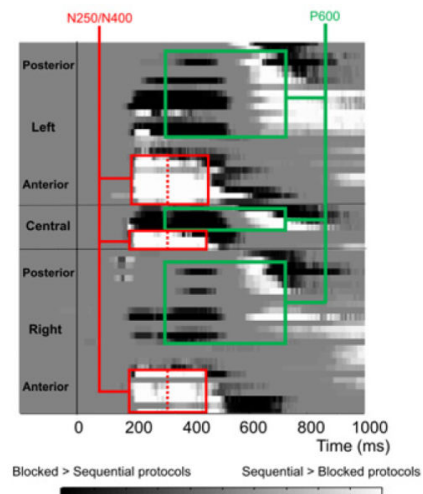

- Sequential naming protocol - Blocked naming protocol

Figure 1.

Event related potentials and statistical test of the contrast between naming protocols. (A) ERPs for the two protocols. For the sake of clarity, only 32 electrodes from among the 60 that were recorded are presented, with a highlight of two representative electrodes showing significant differences $(\mathrm{p}<.001)$. (B) Results of running Student t-tests comparing naming protocols performed for every electrode. The tests were iterated over multiple sub-samples of the data to take into account differences in the size of the data sets across protocols; the scale indicates the proportion of sub-samples for which there was a significant difference ( $p$ $<.001$ ) in either direction (see Methods for details). Red squares delineate the earliest significant differences, interpreted as N 250/N 400 components. Green squares delineate later significant differences. 

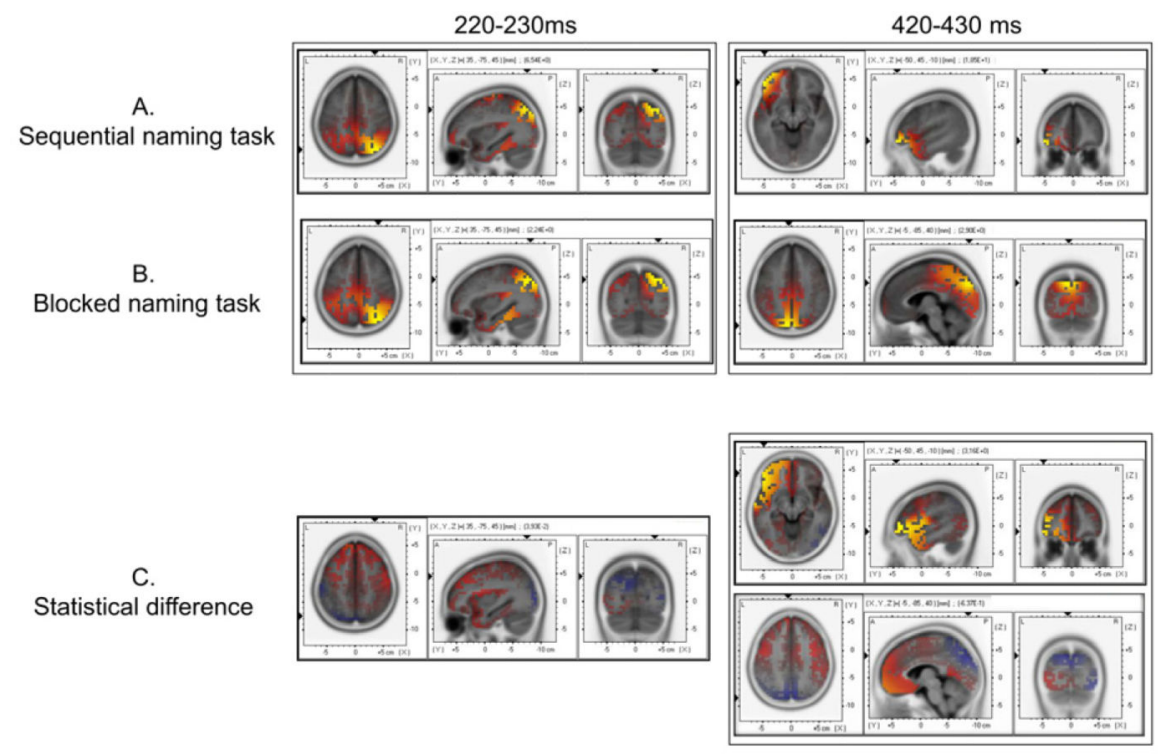

Figure 2.

sLORETA localization of the electrophysiological activity of the grand average for the sequential (A) and the blocked (B) naming protocols at 220-230 ms (top-left) and 420-430 ms (top-right) post-stimulus. (C) sLORETA statistical difference between conditions (based on the localizations computed for each participant in each protocol) for the same time windows and regions of interest. The differences observed were marginally significant across participants ( $\mathrm{p}=.08$ for $220-230 \mathrm{~ms} ; \mathrm{p}=.07$ for $420-430 \mathrm{~ms}$ ). Voxels in red correspond to stronger activity in the sequential naming protocol; voxels in blue correspond to stronger activity in the blocked naming protocol. Within each view, black arrows on the margins indicate the location of the two other views. 
A.

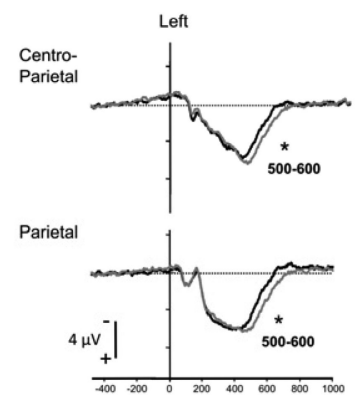

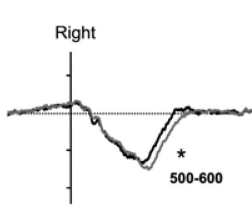

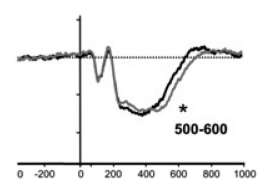

B.

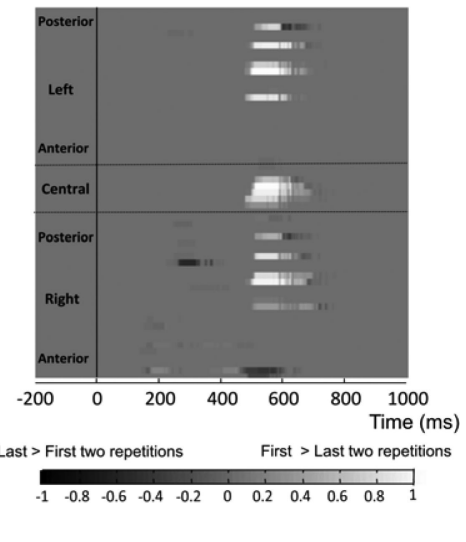

- First two repetitions
- Last two repetitions

Figure 3.

Event related potentials and statistical test of the repetition effect within the blocked naming protocol. (A) ERPs contrasted for the first two and the last two repetitions on CP1, and P3 (left hemisphere), and CP2, P4 (right hemisphere). Significant effect of repetition noted. The star denotes a significant effect of repetition. (B) Running Student t-test results for all electrodes comparing the first and the last two repetitions within the blocked naming protocol, represented as in Figure 1. 


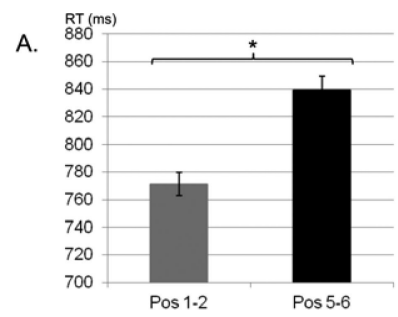

B.
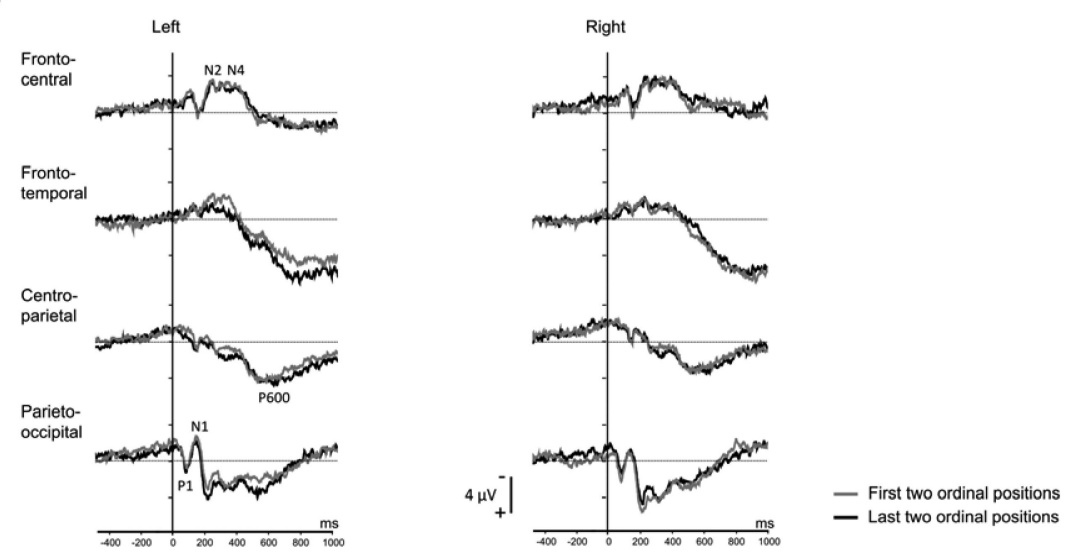

Figure 4.

Semantic contrasts in the sequential naming protocol. (A) Average naming latencies in milliseconds for the first two and the last two ordinal positions. A significant semantic interference effect is observed (see also Figure S2). (B) Event related potentials in the sequential naming protocol, with those from the first two and the last two ordinal positions contrasted, on fronto-central, fronto-temporal and centro-parietal and parieto-occipital electrodes of both hemispheres (FC1, FT7, CP1, PO3 and FC2, FT8, CP2, PO4 respectively). There was no semantic effect (i.e., significant difference between the two ordinal position conditions; see also Figure S3). 


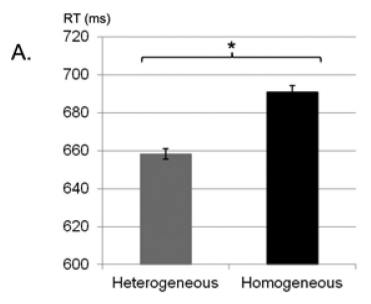

B.
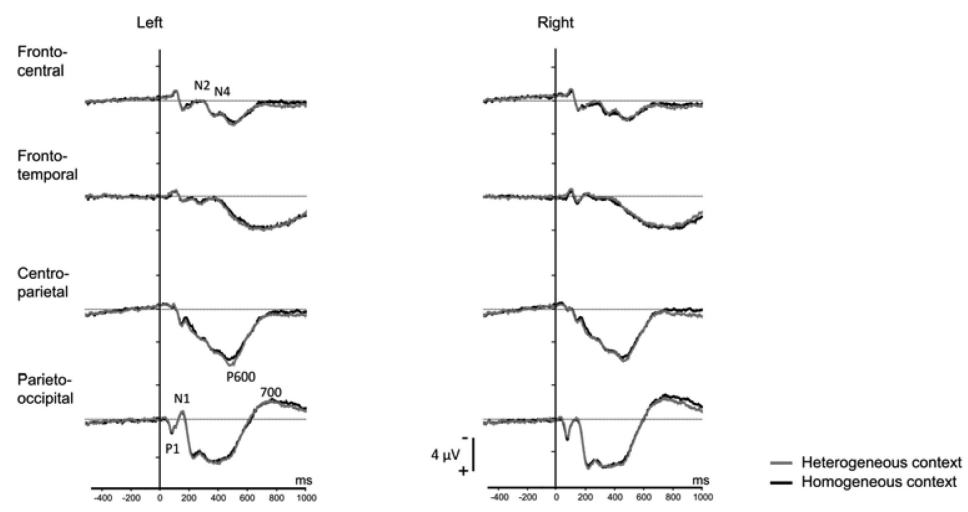

Figure 5.

Semantic contrasts in the blocked naming protocol. (A) Average naming latencies in the two contexts. A significant semantic interference effect is observed (see also Figure S2). (B) ERPs contrasted for homogeneous and heterogeneous blocks, on electrodes FC1, FT7, CP1, and PO3 (left hemisphere), and FC2, FT8, CP2, PO4 (right hemisphere). There was no effect of block homogeneity. 